\title{
Liquid Democracy - eine Realisierung deliberativer Hoffnungen? Zum Selbstverständnis der Piratenpartei
}

\author{
Sebastian Buck
}

Der Erfolg der Piratenpartei seit ihrer Gründung 2006 kann aktuell anhand von zumindest drei Dimensionen verdeutlicht werden. Zunächst ist eine geographische Ausbreitung aus dem großstädtischen Raum in die Flächenländer gelungen und damit die Erwartung widerlegt, es handle sich um ein lokales Phänomen mit einer auf die Ballungsräume begrenzten Zielgruppe. Ein zweiter Faktor ist die steigende Mitgliederzahl in allen Landesverbänden, so dass die Partei bundesweit mittlerweile etwa 30.000 Mitglieder umfasst (Stand: April 2012), von denen rund 18.000 erst nach dem Jahresbeginn 2011 eingetreten sind. ${ }^{1}$ Am wirkmächtigsten fallen jedoch die Erfolge bei den verschiedenen Landtagswahlen (Berlin: 8,9 Prozent; Saarland: 7,4 Prozent; Schleswig-Holstein: 8,2 Prozent; Nordrhein-Westfalen: 7,8 Prozent) ins Gewicht.

Aus demokratietheoretischer Perspektive ist vor allem das Selbstverständnis der Piratenpartei und ihr Versprechen interessant, Politik ,anders“ zu organisieren. Liquid Democracy als Konzept der parteiinternen Willensbildung soll die politische Partizipation der Bürger stärken. Diese soll zu einem permanent wirksamen Faktor im politischen Alltag werden, ohne ein politisches Handeln der Verantwortlichen zu verhindern. Dahinter steht die Vision, Politik trotz eines hohen Spezialisierungsgrads der von ihr zu lösenden Aufgaben nicht in der Abhängigkeit vom Expertentum zu belassen ${ }^{2}$, sondern auch inhaltlich und moralisch komplexe Fragen an die Bürger zurückzubinden.

\section{Liquid Democracy und Liquid Feedback}

Die Entwicklungsgeschichte von Liquid Democracy verlief nicht linear. Vielmehr ist sie das Ergebnis eines Diskurses mit losen Enden, der seit etwa 50 Jahren geführt wird und nur eingeschränkt von der Wissenschaft getragen und dokumentiert wurde. Ausgangspunkt waren erste Entwicklungen im Bereich des Internets in den 1960er Jahren und die mit dem neuen Medium verbundene Aussicht, Informationen, Meinungen und Entscheidungen einer de facto unbegrenzten Zahl von Menschen in Sekundenbruchteilen zu Ergebnissen zusammenzuführen.

Mit James C. Miller wurden diese Potentiale in ein erstes Programm zur Gestaltung politischer Prozesse gefügt. Im Zentrum stand die Möglichkeit der Wähler, sich ihre Repräsentanten auszusuchen, diese bei Bedarf zu wechseln oder über Sachverhalte selbst abzustim-

1 Vgl. Piratenwiki, Mitglieder, https://wiki.piratenpartei.de/Mitglieder\#Mitgliederentwicklung (Abruf am 2. Juli 2012). Das Piratenwiki wird seitens der Piratenpartei als zentrale Informations- und Koordinationsplattform betrachtet, was die häufigen Verweise auf die Plattform erklärt. Der im vorliegenden Text hohe Anteil an Internetquellen ist dem Umstand geschuldet, dass die angegebenen Texte überwiegend über dieses Medium veröffentlicht werden.

2 Vgl. Alexander Bogner / Wolfgang Menz, Wissenschaftliche Politikberatung? Der Dissens der Experten und die Autorität der Politik, in: Leviathan, 30. Jg. (2002), H. 3, S. 384 - 399, S. 385. 
men. ${ }^{3}$ Die Repräsentanten wurden so zu „Proxys “4 , deren Stimmgewicht genauso groß ist, wie die Anzahl an Wahlberechtigten, die sie momentan vertreten. Als Proxys sind Repräsentanten in der Ausübung ihres Mandats frei; jedoch sind die an sie delegierten Stimmen nicht für einen festen Zeitraum, etwa eine Wahlperiode, übertragen. Das Stimmgewicht der Repräsentanten kann folglich von Abstimmung zu Abstimmung variieren.

Mit Beginn der 2000er Jahre wurde der Begriff Liquid Democracy in seinem heutigen Verständnis geprägt, dies vor allem in Internetforen und Internetcommunities. Der Diskurs griff dabei unsystematisch auf verschiedene Proxy- und Delegationsmodelle zurück ${ }^{5}$ und wurde von der Vorstellung getragen, grundlegende demokratische Normen zu realisieren. ${ }^{6}$

Die Piratenpartei hat Liquid Democracy nicht bereits seit ihrer Gründung als das zentrale Medium zur Generierung politischer Entscheidungen bestimmt. Erst 2007 begannen Diskussionen um das Konzept. Liquid Feedback ist das Ergebnis dieser Diskussionen und operationalisiert seit 2009 Liquid Democracy für die Piratenpartei. ${ }^{7}$

Es versteht sich als Möglichkeit, direkte und repräsentative Demokratie miteinander zu verschränken. Anspruch der Betreiber ist es, dem Meinungsbildungsprozess einen Rahmen zu geben, der ein hohes Rationalitätsniveau erwarten lässt und auf dessen Grundlage eine Abstimmung erfolgen kann. ${ }^{8}$ Bis eine Initiative beschlossen und damit die Meinung der Plattformnutzer dargestellt ist, muss sie immer folgende Schritte durchlaufen:

\section{Abbildung 1: Stufen im Liquid Feedback der Piratenpartei}

\begin{tabular}{|c|c|c|c|}
\hline \multicolumn{2}{|c|}{ Quorum } & \multicolumn{2}{c|}{ Quorum } \\
\hline Neu & Diskussion & Eingefroren & Abstimmung \\
\hline
\end{tabular}

Quelle: Lars Reinke, Video-Präsentation vom 3. März 2012, http://larsreineke.de/liquidfeedback-vortrag/ (Abruf am 2. Juli 2012).

3 Vgl. James Miller, A Program for Direct and Proxy Voting in the Legislative Process, in: Public Choice, 7. Jg. (1969), H. 1, S. 107 - 113, S. 108.

4 Die maßgebliche Prägung des Begriffs erfolgte durch William S. U'Ren. Vgl. Oregon Blue Book, Initiative, Referendum and Recall: 1912-1914, Verfassungsänderung Nr. 31 vom 5. November 1912, http://bluebook.state.or.us/state/elections/elections12.htm (Abruf am 2. Juli 2012); Government By Proxy Now. Oregon Plan Would Present Ideas of Representative Lawmaking, in: New York Times vom 29. Juni 1912, http://query.nytimes.com/mem/archive-free/pdf?res=980CEED 91E3CE633A25753C3A9609C946396D6CF (Abruf am 2. Juli 2012).

5 Vgl. zum Beispiel Bryan Ford, Delegative Democracy, 15. Mai 2002, S. 9, http://www.brynosaurus.com/deleg/deleg.pdf (Abruf am 2. Juli 2012).

6 Proxys als Delegationsmodelle werden neben der Informatik auch in den Wirtschaftswissenschaften zur Beschreibung gesellschafts- und aktienrechtlicher Modelle verwendet, siehe zum Beispiel Ekkehart Boehmer, Business Groups, Bank Control, and Large Shareholders: An Analysis of German Takeovers, in: Journal of Financial Intermediation, 9. Jg. (2000), H. 2, S. 117 - 148. James Miller selbst lehnt sich mit seinem Konzept an die mathematischen Schemata von Gordon Tullock (Toward a Mathematics of Politics, Ann Arbor 1967) an; vgl. ders., a.a.O (Fn. 3), S. 113.

7 Vgl. Andreas Nitsche, Interaktive Demokratie durch „Liquid Democracy“, http://liquidfeedback. org/mission/mission-de/ (Abruf am 2. Juli 2012). Weitere Operationalisierungen sind zum Beispiel Adhocracy oder Votorola; eine Übersicht existierender Projekte in diesem Bereich bietet Thomas von der Elbe, Software-Vergleich, http://u.zelea.com/w/User:ThomasvonderElbe_GmxDe/Softwarevergleich (Abruf am 2. Juli 2012).

8 Vgl. Piratenwiki, Liquid Democracy, http://wiki.piratenpartei.de/Liquid_Democracy (Abruf am 2. Juli 2012). 
Sowohl bei der Entscheidung darüber, ob eine Vorlage in die Diskussionsphase übergeht, als auch bei der Frage, ob sie zur Abstimmung gestellt wird, liegt das Quorum bei zehn Prozent derjenigen Plattformmitglieder, die sich für den thematischen Bereich, dem eine Initiative zugeordnet wurde, als Interessenten angemeldet haben. Die Bedingung bezieht sich also nicht auf die Gesamtheit aller Plattformmitglieder. ${ }^{9}$ Unter Rückgriff auf das Proxy-Modell ergeben sich in Abstimmungsprozessen für den Einzelnen folgende Handlungsoptionen:

- Die Teilnahme an der Abstimmung ist möglich, ohne zuvor an Diskussionen teilgenommen zu haben.

- Umgekehrt muss bei einer Teilnahme an der Diskussion keine Teilnahme an der Abstimmung erfolgen.

- Die Abstimmung kann direkt durch den Teilnehmer erfolgen.

- Alternativ kann er seine Stimme einem Delegierten übertragen, wobei jeder Delegierte so viel Einfluss geltend machen kann, wie er Bürger vertritt.

- Delegierte werden erst im Zuge der Stimmübertragung in diesen Status erhoben.

- Einmal getätigte Entscheidungen und Delegationen können bis zum Ende des Entscheidungszeitraums revidiert werden.

Diese Optionen lassen sich auf zwei zentrale Punkte reduzieren, die den „flüssigen“ Aspekt der Liquid Democracy offensichtlich machen: die Flexibilität der Delegation in gleich mehreren Dimensionen sowie die permanente Offenheit für neue Themen, die durch Regeln institutionalisiert werden. Grundlage dieses Verfahrens sind wechselseitige Anerkennung und Vertrauen der Teilnehmer, das Vorhandensein sozialer Beziehungen und der Anreiz zur Vergrößerung des individuellen Bekanntheitsgrads. ${ }^{10}$ Zudem zeichnet sich Liquid Democracy durch eine Vorläufigkeit der auf diesem Wege getroffenen Entscheidungen aus, die nur solange gelten, wie sie nicht auf Grundlage neuer Erkenntnisse und Abstimmungsprozesse geändert werden. ${ }^{11}$

\section{Demokratietheoretische Verortung von Liquid Democracy}

\subsection{Keine Einordnung in die klassischen Demokratiemodelle möglich}

Direkt- oder radikaldemokratische Beteiligungsformen zeichnen sich durch die uneingeschränkte Berücksichtigung des Willens des Bürgers aus, entweder in direkter Abstimmung oder, in ihrer Ausformung als Räterepublik, durch ein imperatives Mandat. ${ }^{12}$ In der Liquid Democracy dagegen handelt der Delegierte zwar auch im Sinne der Delegierenden, da er im Vorfeld eine eigene Position zu einem Thema festlegen muss und erst auf dieser Grundlage das Mandat erhält. Eine spontane Änderung seiner inhaltlichen Position würde ihn gegebenenfalls aus der Rolle des stellvertretenden politischen Akteurs herauslösen und zu einer

9 Vgl. Piratenwiki, Liquid Feedback/FAQ, Punkt 1.2.2 „Was bringt es, Mitglied eines Themenbereichs zu werden?“, http://wiki.piratenpartei.de/LiquidFeedback/FAQ (Abruf am 2. Juli 2012).

10 Vgl. Paolo Boldi / Francesco Bonchi / Carlos Castillo / Sebastiano Vigna, Viscous Democracy For Social Networks, in: Communications of the ACM, 54. Jg. (2011), H. 6, S. 129 - 137, S. 131.

11 Vgl. Piratenwiki, a.a.O. (Fn. 8): „Andererseits können die Piraten ohne Gesichtsverlust auch ihre Meinung ändern, sollten neue Erkenntnisse zu einer neuen Beurteilung des Sachverhalts führen."

12 Vgl. Hans-Joachim Lauth, Regimetypen: Totalitarismus - Autoritarismus - Demokratie, in: ders. (Hrsg.), Vergleichende Regierungslehre, Wiesbaden 2006, S. 91 - 112, S. 95. 
umgehenden Reduzierung seiner Stimmenmacht führen. ${ }^{13}$ Jedoch liegt formal kein imperatives Mandat vor.

Die Unterschiede zu einem repräsentativen System sind ebenso offensichtlich: Den institutionalisierten und formal festgelegten Rollen der Abgeordneten und des Parlaments als Treuhänder (Trustees) werden in der Liquid Democracy vergleichsweise flexiblere Strukturen entgegengesetzt. Diese führen dazu, dass ein Mandat anders als im klassischen Abgeordnetenmodell auch nach der Stimmübertragung nicht unabhängig von den Präferenzen der Delegaten ausgeübt werden kann. Stattdessen werden die Delegierten eng an einen diskursiv erzeugten Willen gebunden, da im Falle eines Abweichens die ihnen übertragenen Stimmen unmittelbar wieder entzogen werden können.

Der zentrale Unterschied gegenüber beiden klassischen Repräsentationsformen besteht in dem Umstand, dass die Liquid Democracy keines zeitaufwendigen, eigens institutionalisierten und formalisierten Kandidatur- und Auswahlverfahrens bedarf, bei dem verschiedene Anwärter um die Rolle eines Abgeordneten konkurrieren. Jeder Bürger wird automatisch zu einem Delegierten für diejenigen, die ihm in einer Sache ihr Stimmrecht übertragen. So soll das Ziel verwirklicht werden, die Responsivität des politischen Systems gegenüber der Öffentlichkeit zu erhöhen.

\subsection{Liquid Democracy als Teil deliberativer Demokratie}

Obwohl sich Liquid Democracy nicht in die zwei großen Modelle politischer Willensbildung einordnen lässt, stellt das Konzept keine Neuerung im demokratietheoretischen Sinne dar. Unabhängig von aktuellen technischen Ausprägungen fallen signifikante Ähnlichkeiten zu deliberativen Demokratietheorien und -konzeptionen auf.

Die deliberative Demokratietheorie umfasst allerdings selber kein homogenes Konzept. In ihrer Genese speist sie sich gleichermaßen aus liberalen wie aus republikanischen Einflüssen. ${ }^{14}$ Die in der Vergangenheit intensiv geführte Debatte, ob der deliberative Kern liberal oder kommunitaristisch sei, verstellt häufig den Blick für ihr Potential, Lösungswege für Sachfragen und moralische Probleme erzeugen zu können. Dieses Potential folgt aus ihrer Konstruktion als eine Theorie zweiter Ordnung, die im Gegensatz zu Theorien erster Ordnung (zum Beispiel Utilitarismus, Libertarismus) die Unausweichlichkeit moralischer Konflikte akzeptiert und unter dieser Voraussetzung nach geeigneten Strategien im Umgang mit begründeten Meinungsverschiedenheiten sucht. ${ }^{15}$

13 Vgl. Norbert Kersting, Innovative Partizipation: Legitimation, Machtkontrolle und Transformation. Eine Einführung, in: ders. (Hrsg.), Politische Beteiligung: Einführung in dialogorientierte Instrumente politischer und gesellschaftlicher Partizipation, Wiesbaden 2008, S. 11 - 39, S. 22.

14 Vgl. Jürgen Habermas, Faktizität und Geltung. Beiträge zur Diskurstheorie des Rechts und des demokratischen Rechtsstaates, Frankfurt am Main 1992, S. 360 f.; ähnlich Maeve Cooke, Five Arguments for Deliberative Democracy, in: Political Studies, 48. Jg. (2000), H. 2, S. 947 - 969 , S. 968.

15 Vgl. Amy Gutmann / Dennis F. Thompson, Why Deliberative Democracy?, Princeton 2004, S. $126 \mathrm{f}$. Theorien erster Ordnung scheitern in ihrem Bemühen, durch eindeutige Paradigmen und Priorisierung einzelner Güter moralischen Konflikten den Raum zu nehmen: „But legitimate moral differences often remain, differences that cannot be resolved without rejecting some of the first-order moral theories mentioned above." (ebenda, S. 129). 
Als Kernelement deliberativer Konzepte, ist der Diskurs der Bürger untereinander über einen Beratungsgegenstand zu nennen. Im Rahmen dieser Form der Konfliktlösung wird durch den Austausch intersubjektiv nachvollziehbarer Argumente das Rationalitätsniveau erhöht. So sollen, ohne vermittelnden oder filternden Eingriff zusätzlicher Akteure, die politischen Einstellungen auf Grundlage zusätzlicher Informationen und höherwertiger Argumente entweder gewandelt oder verfestigt werden. Im Zuge einer möglichen Abstimmung über einen Beratungsgegenstand werden dann nur noch die rational bereinigten Präferenzen erfasst, womit sich deliberative von anderen aggregativen Konstruktionen absetzen. Das durch den Austausch von Argumenten erwachsende gegenseitige Verständnis führt nach einer Abstimmung außerdem dazu, dass die Resultate auch von der unterlegenen Seite in einem höheren Maße akzeptiert werden. ${ }^{16}$

Mit der Liquid Democracy sollen genau diese Aspekte - höheres Rationalitätsniveau, wachsendes Verständnis für unterschiedlich, aber rational begründete Positionen sowie die höhere Akzeptanz der Ergebnisse - erreicht werden. Daher kann die Liquid Democracy als Teil deliberativer Demokratietheorie betrachtet werden.

\subsection{Verortung im deliberativen Spektrum}

Durch die Debatte um den Ursprung der deliberativen Demokratietheorie wird oftmals der Fokus von Kriterien fortgelenkt, die jenseits der normativen Frage nach dem liberalen oder kommunitaristischen Kern eine deskriptiv-analytische Unterscheidbarkeit verschiedener deliberativer Theorieansätze ermöglichen. Gutmann und Thompson haben hierzu ein dichotomes Schema vorgelegt, mit dessen Hilfe die Liquid Democracy im deliberativen Spektrum verortet werden kann. ${ }^{17}$

Eine zentrale Gemeinsamkeit von Liquid Democracy und deliberativer Demokratietheorie stellt die Annahme dar, durch den Diskurs Ergebnisse hervorbringen zu können, die nicht-deliberativen Verfahren potentiell überlegen oder zumindest besser begründbar sind. ${ }^{18}$ Sofern sich eine Theorie vor allem als Instrument zur Herbeiführung deliberativer Ergebnisse versteht, entspricht dies nach Gutmann / Thompson einer instrumentellen Ausprägung von deliberativer Demokratietheorie. Dem steht ein expressiv geprägtes Verständnis gegenüber, bei dem Deliberation über das Erzielen von Ergebnissen hinaus auch eine gemeinschaftsstiftende Funktion hat. ${ }^{19}$ Der letztgenannte Aspekt trifft auf die Konzeption der Liquid Democracy nicht zu. Die angestrebte Beteiligung vieler Personen an Diskursen dient nicht einer zunehmenden Identifikation des Einzelnen mit der Gemeinschaft, sondern vor allem dazu, bessere Ergebnisse zu erzielen. ${ }^{20}$

16 Vgl. Gary S. Schaal / Felix Heidenreich, Einführung in die politischen Theorien der Moderne, Opladen 2009, S. 223 f.

17 Vgl. Amy Gutmann / Dennis F. Thompson, a.a.O. (Fn. 15), S. 21 - 39. Es handelt sich um ein dichotomes Schema mit insgesamt sechs Kriterien, von denen nur drei für das hier behandelte Thema von zentraler Bedeutung sind und daher Verwendung finden.

18 Vgl. Karin Backstrand/ Jamil Khan / Annica Kronsell / Eva Lövbrand, Environmental Politics and Deliberative democracy. Examining the Promise of New Modes of Governance, Cheltenham 2010, S. 3 f.; Gary S. Schaal / Felix Heidenreich, a.a.O. (Fn. 16), S. 23.

19 Vgl. Amy Gutmann / Dennis F. Thompson, a.a.O. (Fn. 15), S. 21 f.

20 Die von der Piratenpartei gewünschte Einbeziehung der Schwarmintelligenz steht exemplarisch dafür, dass vor allem die Qualität der Resultate im Vordergrund steht (vgl. Piratenpartei Nieder- 
Während einige deliberative Verfahren und Theorien die Herausbildung eines Konsenses über den Beratungsgegenstand anstreben, löst die Liquid Democracy Konflikte durch abschließende Abstimmungen. Dieses pluralistische Demokratieverständnis teilt sie mit der Mehrzahl deliberativer Theorien. ${ }^{21}$ Der Aggregation von Einzelmeinungen geht jedoch stets ein verständnis- und kenntniserweiternder Diskurs voraus.

Das Selbstverständnis von Liquid Democracy, alle auch noch so umstrittenen Themen behandeln zu können, führt zu einer starken Betonung formaler Regeln, getakteter Abläufe und Benchmarks, die sowohl den Kern des Konzepts als auch seiner Operationalisierungen wie Liquid Feedback bilden. In der Konsequenz basieren liquide Demokratieprozesse auf einem sehr komplexen Regelwerk. Nur so können die in der Theorie aufgestellten Ziele und Ansprüche in der Praxis realisiert werden. Liquid Feedback benötigt beispielsweise neben einer ausführlichen Liste häufig gestellter Fragen (FAQ-Liste) eine Aufstellung über die Verfahrens- und Abstimmungsmodalitäten bei insgesamt zehn unterschiedlichen Konstellationen je nach Art der Initiative. ${ }^{22}$ Liquid Democracy kann daher als prozeduralistische Theorie verortet werden. ${ }^{23}$

Sie nimmt dennoch für sich in Anspruch, ein praktikables Delegationsmodell zu sein, auf dessen Grundlage Selbstregierung durch „kommunikativ verflüssigte Souveränität“ im Sinne von Jürgen Habermas erreicht werden kann. ${ }^{24}$ Die Selbstregierung der Bürger bedarf zu ihrer Realisierung zweier zentraler Elemente: der Fähigkeit zur Inklusion ihrer Mitglieder und der Rationalität im Diskurs.

Letztere, verstanden als Austausch von Argumenten, die intersubjektiv nachvollzogen werden können, ist im Rahmen der Liquid Democracy, verstanden als ein Teil der deliberativen Demokratietheorie, grundsätzlich herstellbar. Die Operationalisierung durch Liquid Feedback zielt maßgeblich darauf ab, eine Situation zu erzeugen, die einen diese Anforderung erfüllenden Diskurs befördert.

Das Merkmal der Inklusion bedarf hingegen einer detaillierteren Auseinandersetzung. Unter den dargestellten Eindrücken, wonach Liquid Democracy instrumentell ausgerichtet und prozedural konzipiert ist, besteht die Möglichkeit, dass das Konzept jenseits der theoretischen Beteiligungsmöglichkeiten nur wenige Elemente zur Inklusion der Bevölkerung in sich trägt.

sachsen, Piraten in Nordrhein-Westfalen: von 2,0 auf 7,8 Prozent in zweieinhalb Jahren, http:// www.piratenpartei-niedersachsen.de/aktuelles/news/article/piraten-in-nordrhein-westfalen-von-2auf-78-prozent-in-zweieinhalb-jahren.html (Abruf am 2. Juli 2012)).

21 Vgl. Amy Gutmann / Dennis F. Thompson, a.a.O. (Fn. 15), S. 26 f. Als Beispiel für ein konsensuell angelegtes Verfahren kann auf die Planungszelle nach Peter C. Dienel (Die Planungszelle. Der Bürger als Chance, Wiesbaden 2002) verwiesen werden.

22 Vgl. Piratenwiki, a.a.O. (Fn. 9); Piratenpartei, Regelwerke, https://qfb.piratenpartei.de/pp/policy/ list.html (Abruf am 2. Juli 2012).

23 Vgl. Amy Gutmann / Dennis F. Thompson, a.a.O. (Fn. 15), S. 24. Gäbe es zusätzlich zu den formulierten Regelwerken weitere Elemente, die Einfluss auf den Diskurs und seine Ergebnisse nehmen, würde das Verfahren zu den substantiellen deliberativen Demokratietheorien gehören.

24 Vgl. Winfried Thaa, Einleitung, in: ders. (Hrsg.), Inklusion durch Repräsentation, Baden-Baden 2007, S. 9 - 18, S. 11. Auf die Arbeiten von Habermas wurde auch bei der Konstruktion von Liquid Democracy-Verfahren Bezug genommen (vgl. Michael Allen, The Structuring of Power and the Composition of Norms by Communicative Assent, http://zelea.com/project/votorola/d/theory.xht (Abruf am 2. Juli 2012)). 


\section{Liquid Democracy - Fehlen von Inklusionsgedanken}

Das Konzept der Inklusion ist vor allem in substantiellen Demokratietheorien ${ }^{25}$ von großer Bedeutung. Den Kerngedanken bildet dabei die Einsicht, dass nicht Partizipation, sondern Inklusion den tieferen Sinn und Wert von Demokratie darstellt. ${ }^{26}$ Unter Inklusion werden dabei jene Mechanismen verstanden, „die dafür sorgen, dass und wie Personen für relevant gehalten werden [...]“27. Ausgeprägte Inklusivität ist insofern als Grundvoraussetzung für substantielle politische Gleichheit zu verstehen, durch die die Bürger in die Lage versetzt werden, bestehende Partizipationsformen auch tatsächlich wahrzunehmen. ${ }^{28}$ In repräsentativen Systemen geschieht dies beispielsweise über die Wahlkreisabgeordneten und ihre Aufgabe, auch die Interessen jener Bürger zu berücksichtigen, die sie nicht oder gar nicht gewählt haben.

Die genaue Betrachtung von Liquid Democracy und Liquid Feedback als Beispiel ihrer Umsetzung enthüllt zwei Faktoren, die die Inklusionsleistung des Konzepts in Frage stellen. Zusammengenommen führen sie zu der Erkenntnis, dass die Liquid Democracy Inklusion mit Partizipationsmöglichkeiten gleichsetzt und aus diesem Grund einen Mangel an Inklusivität nicht als eigenes Problem wahrnehmen kann.

\subsection{Einschränkungen des rationalen Online-Diskurses}

Der erste Faktor, der eine Inklusion in der Liquid Democracy verhindert, besteht in ihrer ausschließlichen Fokussierung auf den rationalen Diskurs. Zwar entspricht es dem Kern deliberativer Theorien, durch den Austausch rationaler und damit intersubjektiv nachvollziehbarer Gründe zu einem allgemein akzeptierten Ergebnis zu kommen ${ }^{29}$; ausgeblendet wird in der Liquid Democracy jedoch der Umstand, dass neben Rationalität auch Emotionen und persönliche, statistisch nicht signifikante Einzelerfahrungen einen Teil von Argumentationen bilden. ${ }^{30}$ Liquid Democracy bietet keine Ansätze, wie diese Kommunikationsformen in den Diskurs integriert werden können.

Verstärkt wird diese Einschränkung durch die ortsungebundene und virtuelle Diskursführung, in deren Folge dem demokratischen Prozess seine „physische Verortung“ genommen wird. An die Stelle einer über viele Sinne erfahrbaren Demokratie in Versammlungsräumen und Diskussionen tritt eine zumeist rein textbasierte Kommunikation. Die elektronische Übermittlung der Texte erschwert die Deliberation deutlich gegenüber dem persönlichen Kontakt ${ }^{31}$ : Gestik, Mimik, Stimmlage, Erscheinungsbild und weitere

25 Zur Unmöglichkeit wertfreier Demokratiekonzeptionen und dem Verhältnis prozeduraler und substantieller Elemente vgl. Amy Gutmann / Dennis F. Thompson, a.a.O. (Fn. 15), S. 103, S. 108 ff.

26 Vgl. Winfried Thaa, a.a.O. (Fn 24), S. 11.

27 Armin Nassehi, Die Theorie funktionaler Differenzierung im Horizont ihrer Kritik, in: Zeitschrift für Soziologie, 33. Jg. (2004), H. 2, S. 98 - 118, S. 111 (Fn. 21).

28 Vgl. Gary Schaal / Felix Heidenreich, Quality versus Equality?, in: Österreichische Zeitschrift für Politikwissenschaft, 36. Jg. (2007), H. 1, S. 23 - 38, S. 27.

29 „Das Herzstück deliberativer Politik besteht nämlich aus einem Netzwerk von Diskursen und Verhandlungen, das die rationale Lösung pragmatischer, moralischer und ethischer Fragen ermöglichen soll [...].“ Jürgen Habermas, a.a.O. (Fn. 14), S. 388 f.

30 Vgl. Iseult Honohan, Civic Republicanism, London / New York 2002, S. 226.

31 Vgl. Paolo Boldi / Francesco Bonchi / Carlos Castillo / Sebastiano Vigna, a.a.O. (Fn. 10), S. 131. 
Faktoren können nicht zum Transport von Informationen und Intentionen genutzt werden. ${ }^{32}$

De facto erfolgt so eine doppelte Einschränkung: Nach der Beschränkung, was vermittelt werden darf (intersubjektiv nachvollziehbare und rationale Argumente), wird auch das Wie bewusst auf das Textuelle limitiert. Damit verschließt sich die Liquid Democracy grundlegenden Kommunikationsformen und erzeugt eine Hürde bei der Wahrnehmung der gebotenen Partizipationsmöglichkeiten. Unter diesen Beschränkungen leiden zusätzlich die gängigen Identifikationsmöglichkeiten mit Personen und Symbolen demokratischer Institutionen, denen eine hohe inkludierende Wirkung zugeschrieben wird. ${ }^{33}$

\subsection{Auflösung einer gemeinsamen Diskursarena}

Der zweite Faktor für eine mangelnde Inklusivität liegt in der Struktur der Liquid Democracy selbst. Unter anderem durch die Betrachtung der bestehenden Regularien von Liquid Feedback wird deutlich, dass es nicht das Ziel der Liquid Democracy ist, alle Bürger oder Plattformmitglieder bei jeder Thematik zu erreichen. Dies tritt nicht nur im Delegationsmodell zutage, das Bürgern die Möglichkeit einräumt, politische Verantwortung im Zuge der Delegation dauerhaft und vollständig abzutreten. Auch bei der Deutung und Bestimmung der Bezugsgröße für Quoren zeigt sich die Tendenz, nur die in der Liquid Democracy aktiven Bürger zu berücksichtigen: Grundgesamtheiten zur Messung von Quoren oder Entscheidungen umfassen bei Liquid Feeback immer nur die Anzahl von Nutzern, die sich ausdrücklich als an diesem Politikfeld Interessierte gemeldet haben. Sie stellen somit stets nur eine eingeschränkte Grundgesamtheit dar. Die Repräsentativität der auf dieser Basis getroffenen Entscheidungen ist zumindest zweifelhaft.

Problematischer ist aber die Tatsache, dass es überhaupt themenbezogene und damit unterschiedliche Grundgesamtheiten gibt. Die in deliberativen, direktdemokratischen wie auch repräsentativen Systemen bestehende politische Arena aller Bürger wird gar nicht erst als eine Einheit geschaffen, sondern über das Delegationsmodell und themenbezogene Facharenen zersplittert. Für ein neues Thema wird bei Bedarf eine neue Arena geschaffen, in der sich die Interessierten sammeln. ${ }^{34}$ Damit wird der von der Liquid Democracy kritisierte Zustand des politischen Systems auf der Ebene der politischen Öffentlichkeit reproduziert.

In der Liquid Democracy finden sich Fachleute in den themenbezogenen Arenen zusammen, wobei der Diskurs nur von wenigen weiteren Plattformmitgliedern verfolgt wird. In

32 Vgl. Ulrich Mees, Zum Forschungsstand der Emotionspsychologie - eine Skizze, in: Rainer Schützeichel (Hrsg.), Emotionen und Sozialtheorie - Disziplinäre Ansätze, Frankfurt am Main 2006, S. 104 - 124, S. 107. Siehe auch Frank Nullmeier im selben Band zum Forschungsdefizit in diesem Bereich (Politik und Emotion, S. 84 - 103).

33 Vgl. Achim Hurrelmann, Verfassung und ökologische Krise - Verfassungstheoretische Lösungsansätze für Umweltprobleme, Hamburg 2009, S. 98 (in Anlehnung an Rudolf Smend, Staatsrechtliche Abhandlungen und andere Aufsätze, Berlin 1968).

34 Bei der Piratenpartei und Liquid Feedback erfolgt die Ausdifferenzierung nicht nur thematisch, sondern auch inhaltlich. Exemplarisch wird hier auf parallele Arbeitsgruppen zur Außenpolitik verwiesen, die sich nicht analog zu der Flügelbildung anderer Parteien grundsätzlich unterschiedlich positionieren, sondern bereits bei einem Thema in getrennte Diskursarenen ausweichen (vgl. Annett Meiritz / Raniah Salloum, Stunde der Freaks, http://www.spiegel.de/politik/deutschland/ piraten-ringen-um-positionen-zur-aussenpolitik-a-836801.html (Abruf am 2. Juli 2012)). 
der Tendenz - und dies ist mit Blick auf die Frage der demokratischen Legitimation dieser Beratungsform entscheidend - können einzelne Akteure den Diskurs innerhalb der Kleinarenen massiv beeinflussen, da die Diskursmacht allein überdurchschnittlicher Beteiligung und Aktivität entspringen kann. ${ }^{35}$

Vor dem Hintergrund des beschriebenen Anspruchs demokratischer Arrangements, die Bevölkerung zu inkludieren, ist die Auflösung einer allgemeinen Diskursarena jedoch kritisch zu beurteilen. Hiermit entfällt zwangsläufig die Möglichkeit, in der zentralen Arena die Vielzahl von Fachdiskursen zu bündeln und der tatsächlichen Grundgesamtheit aller Bürger vorzustellen. Wie aber sollen Bürger über die Entwicklung von Diskursen in für sie zunächst fernen Politikfeldern informiert werden? Und wie können jenseits einer persönlichen Betroffenheit oder Neigung Bürger angeregt werden, sich auf neue Themen einzulassen? Liquid Democracy stößt hier offenbar an ihre Grenzen, da sich aus dem dargestellten Konstrukt der Theorie heraus keine Hinweise ergeben, die eine Beantwortung der Frage ermöglichen.

\section{Perspektiven der Liquid Democracy}

Der Erfolg der Piratenpartei hat die mediale und politische Landschaft für ein Phänomen politischer Willensbildungsprozesse geöffnet, das nur in geringem Maße von der Fachdisziplin der Politikwissenschaft erarbeitet oder geprägt wurde. Liquid Democracy als ein flexibles System zur Beteiligung oder Delegation der eigenen Stimme kann dennoch in den bestehenden Kanon deliberativer Konzeptionen eingeordnet werden. Deliberation als Mechanismus zur Gewährleistung größtmöglicher Rationalität zu verstehen, stellt bereits eine Verengung der Bandbreite der Theorie dar. Durch prozedurale Diskursbeschränkungen und die Vielzahl politischer Arenen verliert die Liquid Democracy jedoch ihr inkludierendes Potenzial, das eine Beteiligung von weiten Teilen der Bevölkerung als den eigentlichen und tieferen Sinn von Demokratie anerkennt.

Das Fehlen inkludierender Bestandteile unterstützt die Herausbildung von unabhängigen Facharenen, in denen Experten, analog zu Fachpolitikern und der Arbeit von parlamentarischen Ausschüssen, Sachfragen ohne nennenswerte Beteiligung „von außen“ regeln und Entscheidungen beziehungsweise Meinungsbilder herbeiführen. Eine „fall back“-Option ermöglicht es dem Einzelnen, einen dauerhaften Delegierten zu benennen, unabhängig vom Thema und dessen Position dazu. An die Stelle einer Direktbeteiligung der Bürger treten de facto Repräsentationsformen, die der Komplexität der Sachfragen oder dem mit ihrer Klärung verbundenen Zeitaufwand Tribut zollen. ${ }^{36}$

Repräsentative Systeme bieten demgegenüber eine hohe Inklusionsleistung, da sie allen, auch politisch desinteressierten Bürgern eindeutige Repräsentanten zuweisen und deren Interessen im Rahmen stabiler, persönlicher und ortsgebundener Strukturen berücksichtigen können. Auf der Basis von Vertrauen in die Verlässlichkeit des politischen Systems werden

35 Vgl. Paolo Boldi / Francesco Bonchi / Carlos Castillo / Sebastiano Vigna, a.a.O. (Fn. 10), S. 130 f. Die Autoren erkennen in ihrer Analyse von Online-Diskursen anstelle von deliberativen vielmehr meritokratische Entscheidungsstrukturen. Der Einfluss von „drive-by“-Teilnehmern wird dabei marginalisiert, unabhängig von der rationalen oder argumentativen Güte ihrer Beiträge.

36 Vgl. hierzu die in der Piratenpartei geführte Diskussion um „Superdelegierte“, zum Beispiel MarieKatharina Wagener, Schwarm der Superdelegierten - Inoffiziell hat die Piratenpartei schon ein Vollprogramm, in: FAZ vom 28. April 2012, S. 10. 
weite Teile der Gesellschaft in das System inkludiert, wodurch eine Grundlage für die Akzeptanz herbeigeführter Entscheidungen entstehen kann. ${ }^{37}$

Angesichts des Defizits an Inklusionsmechanismen ist zu bezweifeln, dass in Liquid Democracy ein Ersatz für bestehende repräsentative Strukturen gesehen werden kann. Im Rahmen einer Diskussion um die mögliche Ergänzung des etablierten Systems durch Liquid Democracy-Elemente muss zwangsläufig gefragt werden, wie das Kriterium substantieller politischer Gleichheit gewahrt werden kann. Die Notwendigkeit, sich dieser Debatte zu stellen, haben die politischen Akteure erkannt. So hat der Deutsche Bundestag die Enquete-Kommission „Internet und digitale Gesellschaft " einberufen ${ }^{38}$, welche die Zukunft der digitalen Gesellschaft zum Thema hat und dabei Bürger mittels Adhocracy in die Diskussion einbinden will. ${ }^{39}$

37 Vgl. Enquete-Kommission Internet und Digitale Gesellschaft, Wer treibt den Strukturwandel der politischen Kommunikation und Partizipation voran - und wo bleiben die Anderen?, https://www. bundestag.de/internetenquete/dokumentation/Sitzungen/20120319/A-Drs_17_24_049-B_-_ Stellungnahme_Prof_Dr_Vowe_19_3_2012.pdf (Abruf am 2. Juli 2012), S. 6 f. Die Defizite repräsentativer Arrangements in unterschiedlichen Dimensionen sollen durch diesen Verweis nicht abgeschwächt oder ausgeblendet werden.

38 Enquete-Kommission Internet und Digitale Gesellschaft, Wissen, Ideen und Expertise für die Politik, https://enquetebeteiligung.de/ (Abruf am 2. Juli 2012).

39 Für hilfreiche Hinweise und Kritik einer früheren Fassung des Manuskripts danke ich Prof. Walter Reese-Schäfer und den Teilnehmern seines Doktorandenkolloquiums.

\title{
Parlamentarisches Ordnungsgeld und Sitzungsausschluss: Verfassungsrechtliche Aspekte ${ }^{*}$
}

\author{
Oliver Borowy
}

Der Deutsche Bundestag hat in seiner Sitzung am 30. Juni 2011 beschlossen, sein parlamentarisches Ordnungsrecht um eine weitere Ordnungsmaßnahme, das so genannte Ordnungsgeld, zu ergänzen. ${ }^{1}$ Der sitzungsleitende Präsident ${ }^{2}$ kann nunmehr bei einer nicht nur geringfügigen Verletzung der Ordnung oder der Würde des Bundestages ein Ordnungsgeld in Höhe von 1.000 Euro, im Wiederholungsfall von 2.000 Euro festsetzen. Mit dem Ordnungsgeld soll nach dem Willen des Parlaments eine im Sanktionssystem der Geschäftsordnung vorhandene Lücke geschlossen werden, die zwischen dem Ordnungsruf am unteren Ende und dem Sitzungsausschluss am oberen Ende der Skala besteht, um dem Präsidenten

* Zugleich eine Erwiderung auf Albert Ingold / Sophie-Charlotte Lenski, Ordnungsgeld und Sitzungsausschluss als Ordnungsmaßnahmen gegen Bundestagsabgeordnete, in: JZ, 67. Jg. (2012), H. 3, S. 120 - 125. Der Verfasser ist Mitarbeiter der Bundestagsverwaltung. Der Beitrag gibt allein seine persönliche Auffassung wieder.

1 BT-Plenarprotokoll 17/117 vom 30. Juni 2011, S. 13531 C.

2 Im weiteren Text wird nur vom Präsidenten gesprochen. Gemeint ist damit die jeweils sitzungsleitende Präsidentin beziehungsweise der jeweils sitzungsleitende Präsident, sofern nicht ausdrücklich vom Bundestagspräsidenten die Rede ist. 